\title{
Higher Leaf Area Improves the Productivity of Finger Millet (Eleusine coracana (L.) Gaertn) under Rainfed Conditions
}

\author{
Y.A. Nanja Reddy ${ }^{1,2 *}$, Jayarame Gowda ${ }^{1}$, E.G. Ashok ${ }^{1}$, \\ K.T. Krishne Gowda ${ }^{1}$ and M.V.C. Gowda ${ }^{1}$ \\ ${ }^{1}$ All India Coordinated Small Millets Improvement Project, University of Agricultural \\ Sciences, GKVK, Bangalore 560 065, Karnataka, India \\ ${ }^{2}$ Department of Crop Physiology, University of Agricultural Sciences, GKVK, Bangalore 560 \\ 065, Karnataka, India \\ *Corresponding author
}

K e y w o r d s
LAI, DM/LAD,
Biomass, Grain
yield

\section{A B S T R A C T}

\section{Introduction}

Finger millet is an important staple food crop of southern Karnataka predominantly grown under dry land conditions in light soils with low input, traditionally in cereal based farming systems during monsoon season. Significant yield improvement was achieved over the years, through exploitation of genetic variability for specific traits, such as blast resistance in addition to agronomic manipulations. Presently finger millet occupies an area of 1.2 mha with a production of $2.0 \mathrm{mt}$ in India (Malhotra, 2018). However there is a decreasing trend in area but with an increased productivity (Anon, 2011) and stagnated grain yield (Swetha, 2011), to break this barrier, an approach of physiological traits associated with grain yield would be highly relevant. Breeding efforts have shown yield improvement of rice and wheat through improved HI, while, in maize through biomass (Richards, 2000). Hence, to break the yield plateau in finger millet, identification of accessions for traits associated with high biomass and its efficient partitioning and; 
incorporation of such traits in breeding programmes would be highly effective (Shankar et al., 1990). The present investigation examines the relevance of LAI and net assimilation rate (DM/LAD) towards biomass production and grain yield besides identifying superior finger millet accessions for these traits.

\section{Materials and Methods}

Field experiment was conducted during kharif season of 2007 at GKVK Farm on red sandy loam soil with a $\mathrm{pH}$ of 6.5 . Twenty three accessions and ten varieties were replicated twice in RCBD with a net plot size of 1.44 $\mathrm{m}^{2}$. Crop was managed as per the recommended package of practices (FYM, 7.5 t.ha ${ }^{-1}$ and NPK @ 50:40:25 kg.ha ${ }^{-1}$ ). Rainfall during the cropping season was $806 \mathrm{~mm}$, but experienced a long dry spell of 27 days during September $21^{\text {st }}$ to October $18^{\text {th }} 2007$.

Leaf area and biomass at flowering and; yield attributes at harvest were recorded. The total dry matter/ leaf area duration up to flowering (DM/LAD), as a measure of assimilation rate was computed and expressed as $\mathrm{g} \mathrm{m}^{-2} \mathrm{day}^{-1}$. Extent of contribution of LAI and DM/LAD towards dry matter production was computed using standard partial regression co-efficient arrived through multiple regression analysis. The data was analyzed using MSTAT-C programme and correlations among the parameters were computed.

\section{Results and Discussion}

Wide genotypic variability was observed for yield, yield attributes and physiological traits viz., LAI, assimilation rate (DM/LAD), biomass etc. (Table 1). A similar large variation for these parameters among 400 finger millet germplasm lines was reported by Shankar et al., (1990) and in another study by Aparna and Ansari (2017) wherein maximum
LAI accumulation was observed at 45 DAS. These variations provide an opportunity for selection of trait specific accessions associated with high grain yield (Table 2).

Under adequate input conditions, the crop productivity will be determined primarily by the average canopy cover (LAI), net assimilation rate (DM/LAD) and the crop duration. In the present study, grain yield of mid-duration genotypes was distinctly high (321.9 $\left.\mathrm{gm}^{-2}\right)$ compared long duration (245.4 $\mathrm{g})$ or short $(253.4 \mathrm{~g})$ accessions (Table 1) and; the relationship between duration and grain yield is also not positive $(\mathrm{r}=-0.21$, Table 4$)$. These results are in contrast to the expected direct relationship between duration and grain yield (Bedis et al., 2006), because, the long duration genotypes were caught up with dry spell for 27 days during critical stages viz., flag leaf, ear emergence and $50 \%$ flowering. During these critical stages, the long duration accession received only $60.1 \mathrm{~mm}$ with 5 rainy days compared to $143.9 \mathrm{~mm}$ with 10 rainy days for medium duration accessions (Table 3). Further, long duration accessions coincided with higher soil temperature of 27.3 to $30.2^{0} \mathrm{C}$ at $10 \mathrm{~cm}$ depth compared to 26.2 to $27.9^{\circ} \mathrm{C}$ for medium duration types. Hence, the medium duration varieties are better options in the changing climate scenario with more number or long duration of intermittent moisture stress situations during kharif seasons.

Correlation analysis (Table 4) among various physiological and yield attributing traits is very pertinent to establish selection criteria for yield improvement. In physiological terms grain yield is the product of above ground biomass and partitioning of biomass to ear (HI). Among these two, the biomass was strongly correlated to grain yield $(\mathrm{r}=0.87 * *)$ as compared to the HI $(\mathrm{r}=0.52 * *)$ (Table 4). The multiple regression analysis also showed that the contribution of biomass towards grain 
yield was more (59\%) compared to the HI (41 \%). Further, the biomass at harvest and; grain yield are also positively related to yield components viz., ear number $(\mathrm{r}=0.32,0.30)$, ear weight $\left(\mathrm{r}=0.90^{* *}, 0.95^{* *}\right)$ and test weight $\left(\mathrm{r}=0.45^{* *}, 0.69 * *\right)$. Similar correlations in finger millet have been reported by Udayakumar et al., (1986), Sharathbabu et al., (2008), Nandini et al., (2010) and Wolie and Dessalegn (2011).

The HI showed positive relationship with ear weight $\left(\mathrm{r}=0.38^{*}\right)$, threshing percent $\left(\mathrm{r}=0.63^{* *}\right)$ and test weight $\left(\mathrm{r}=0.66^{* *}\right)$ and not related to LAI $(r=-0.06)$. This indicates that the increase in these yield attributing traits would result in higher grain yield through significant increase in HI. However increase in $\mathrm{HI}$ alone may reduce the biomass investment in leaves and other vegetative structures, loosing total biomass production as evidenced in Cv. GPU-67 (Swetha, 2011). Therefore it is appropriate to focus on ways and means to increase the biomass by maintaining at higher $\mathrm{HI}$ values in finger millet.

The biomass accumulation is determined by both current photosynthates and remobilization of carbohydrates of the stem to ear during reproductive phase. The current photosynthates in turn depend on functional leaf area (LAD), while the remobilization depends upon the biomass available at the time of flowering when the sink is not a limitation. The biomass production by flowering stage in turn depends on light interception which can be manipulated by the LAI, LAD and leaf angle. However, in finger millet the LAI (2.5) is still low (Uma 1987), but has positive and significant correlation with grain yield and biomass $\left(\mathrm{r}=0.32,0.41^{* *}\right.$, Table 4, Kumar et al., 2006 and Sharathbabu et al., 2008). Hence, it appears that LAI is most limiting factor for productivity when net assimilation rate is not a limitation. Further, the leaf area especially with broad leaf is highly inheritable (Richards et al., 2001) and can also be manipulated easily through agronomic approaches such as plant population (Roy et al., 2002), growth regulators (Sujatha and Rao, 2003), nutrition (Khalak and Kumaraswamy, 1994) and weed management (Kumara et al., 2007). Therefore selection of high LAI would result in increased biomass and grain yield of finger millet to break the yield plateau.

The other component of biomass determination, DM/LAD, a measure of net assimilation rate, is poorly related $(r=0.20 \mathrm{NS}$, Table 4), probably finger millet being a $\mathrm{C}_{4}$ NAD-ME species (Siebke et al., 2003) maintain relatively higher photosynthetic rate and has better photosynthates translocation due to dense minor longitudinal veins (Ueno et al., 2006). These results although suggest that, assimilation rate may not constrain the productivity in finger millet, the short duration accessions possess distinctly higher DM/LAD with lower LAI values, hence, these two traits may compensate each other, thus possibilities of breeding for higher NAR cannot be precluded.

Hence of the two parameters, the contribution of LAI towards biomass production is relatively high compared to net assimilation rate / photosynthetic rate as also reported by Vishwanath (2005) and Subrahmanyam (2000).

Further, the LAI was reported to have positive relationship with seed yield (Veeraputhiran et al., 2009; John and Kumar, 2018), but not the single plant leaf area or flag leaf area (Narayan et al., 2018). In the present study also the contribution of LAI towards biomass production at flowering and crop maturity is 69.3 and $65.2 \%$ respectively compared to $\mathrm{DM} / \mathrm{LAD}$ of 30.7 and 34.8 percent respectively. 
Table.1 Physiological parameters at flowering and yield attributes at harvest in finger millet accessions

\begin{tabular}{|c|c|c|c|c|c|c|c|c|c|c|c|c|c|c|c|c|}
\hline $\begin{array}{l}\dot{\mathbf{z}} \\
\dot{\vec{B}}\end{array}$ & 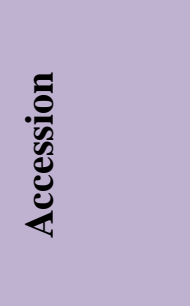 & $\frac{\pi}{0}$ & 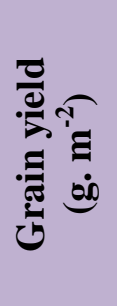 & 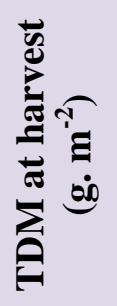 & 玨 & 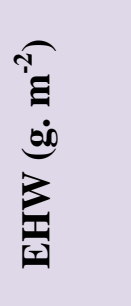 & 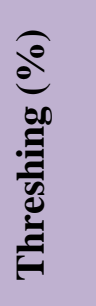 & 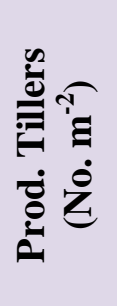 & 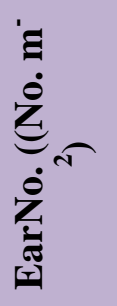 & 司0 & 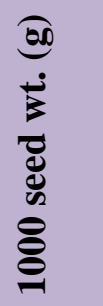 & 寻 & 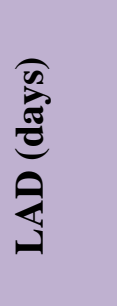 & 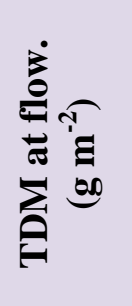 & 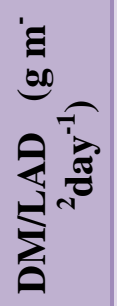 & 胥 \\
\hline 1 & GPU-48 & 68 & 245.9 & 551.9 & 0.45 & 300.0 & 0.82 & 51.1 & 55.6 & 5.39 & 2.53 & 1.82 & 61.7 & 411.8 & 6.67 & 5.96 \\
\hline 2 & Indaf-9 & 62 & 364.9 & 895.3 & 0.41 & 466.3 & 0.80 & 58.4 & 70.2 & 6.60 & 2.83 & 1.90 & 58.9 & 479.0 & 8.28 & 5.93 \\
\hline 3 & VR-708 & 54 & 216.0 & 434.0 & 0.50 & 260.4 & 0.83 & 74.3 & 103.5 & 2.52 & 3.06 & 1.32 & 35.5 & 264.2 & 7.43 & 6.36 \\
\hline 4 & GE-162 & 58 & 240.7 & 607.8 & 0.40 & 324.3 & 0.74 & 90.0 & 89.3 & 3.65 & 2.10 & 3.04 & 88.2 & 555.1 & 6.33 & 5.61 \\
\hline 5 & GE- 1034 & 63 & 277.8 & 622.0 & 0.44 & 333.7 & 0.83 & 48.3 & 52.8 & 6.24 & 2.86 & 2.78 & 87.4 & 592.4 & 6.79 & 5.18 \\
\hline 6 & GE-2770 & 70 & 160.1 & 470.7 & 0.34 & 225.7 & 0.71 & 86.5 & 90.7 & 2.50 & 1.43 & 1.44 & 50.2 & 249.9 & 5.07 & 5.27 \\
\hline 7 & GE-3370 & 59 & 220.8 & 497.8 & 0.45 & 267.4 & 0.83 & 65.3 & 118.1 & 2.29 & 2.56 & 2.45 & 72.3 & 414.6 & 5.86 & 5.28 \\
\hline 8 & GE-4222 & 63 & 325.3 & 719.1 & 0.45 & 378.5 & 0.86 & 90.7 & 113.6 & 3.30 & 3.02 & 2.17 & 68.4 & 408.4 & 6.10 & 5.55 \\
\hline 9 & GE-4732 & 63 & 228.8 & 590.4 & 0.38 & 277.8 & 0.83 & 43.1 & 45.9 & 5.94 & 2.95 & 1.45 & 45.5 & 319.8 & 7.06 & 6.03 \\
\hline \multicolumn{2}{|c|}{ Mean (SD) } & 62 & 253.4 & 598.8 & 0.42 & 314.9 & 0.81 & 67.5 & 82.2 & 4.27 & 2.59 & 2.04 & 63.1 & 410.6 & 6.62 & 5.69 \\
\hline 10 & GPU-28 & 74 & 328.9 & 790.6 & 0.41 & 495.2 & 0.67 & 63.9 & 108.4 & 4.59 & 3.39 & 2.97 & 109.9 & 623.8 & 5.69 & 6.53 \\
\hline 11 & Indaf-7 & 74 & 275.0 & 631.2 & 0.44 & 356.6 & 0.78 & 68.1 & 93.4 & 3.86 & 2.53 & 1.95 & 72.2 & 348.0 & 4.81 & 5.58 \\
\hline 12 & HR-911 & 74 & 307.7 & 697.5 & 0.44 & 363.9 & 0.85 & 61.5 & 70.5 & 5.13 & 2.99 & 2.00 & 74.0 & 331.9 & 4.49 & 5.44 \\
\hline 13 & PR-202 & 73 & 301.8 & 637.5 & 0.47 & 352.8 & 0.86 & 87.9 & 116.0 & 3.04 & 3.13 & 2.48 & 90.5 & 455.4 & 5.05 & 5.34 \\
\hline 14 & GE-619 & 73 & 339.3 & 889.4 & 0.38 & 426.1 & 0.80 & 92.7 & 201.1 & 2.13 & 2.95 & 3.63 & 132.3 & 654.7 & 4.97 & 5.00 \\
\hline 15 & GE-1013 & 74 & 378.5 & 832.7 & 0.46 & 454.8 & 0.84 & 66.0 & 78.9 & 5.88 & 2.68 & 2.51 & 92.9 & 538.7 & 5.80 & 6.30 \\
\hline \multicolumn{2}{|c|}{ Mean (MD) } & 74 & 321.9 & 746.5 & 0.43 & 408.2 & 0.80 & 73.4 & 111.4 & 4.11 & 2.95 & 2.59 & 95.3 & 492.1 & 5.14 & 5.70 \\
\hline 16 & MR-6 & 78 & 365.7 & 814.5 & 0.45 & 446.6 & 0.83 & 63.2 & 72.9 & 6.09 & 3.01 & 3.14 & 122.5 & 516.9 & 4.22 & 5.61 \\
\hline 17 & Indaf-8 & 83 & 269.1 & 610.9 & 0.44 & 343.8 & 0.79 & 66.7 & 67.0 & 5.17 & 2.89 & 2.44 & 101.1 & 301.3 & 2.98 & 5.86 \\
\hline 18 & L-5 & 83 & 247.3 & 522.0 & 0.47 & 306.3 & 0.81 & 51.4 & 59.0 & 5.24 & 3.20 & 1.71 & 70.8 & 359.8 & 5.08 & 6.00 \\
\hline 19 & GE-224 & 82 & 253.8 & 639.7 & 0.40 & 319.8 & 0.80 & 95.1 & 125.7 & 2.56 & 2.01 & 2.35 & 96.1 & 370.7 & 3.89 & 5.28 \\
\hline 20 & GE-844 & 82 & 276.8 & 657.3 & 0.41 & 333.0 & 0.83 & 49.0 & 54.2 & 6.02 & 2.37 & 2.60 & 106.4 & 385.7 & 3.62 & 5.23 \\
\hline 21 & GE-2858 & 82 & 207.3 & 514.3 & 0.40 & 271.6 & 0.77 & 61.5 & 76.1 & 3.57 & 2.45 & 3.21 & 131.4 & 530.3 & 4.04 & 5.36 \\
\hline 22 & GE-3067 & 83 & 233.0 & 575.9 & 0.41 & 301.8 & 0.77 & 72.3 & 77.8 & 3.89 & 1.80 & 2.23 & 92.3 & 413.8 & 4.48 & 4.98 \\
\hline
\end{tabular}




\begin{tabular}{|c|c|c|c|c|c|c|c|c|c|c|c|c|c|c|c|c|}
\hline 23 & GE-3069 & 82 & 190.0 & 584.9 & 0.33 & 266.0 & 0.72 & 61.9 & 87.9 & 3.05 & 1.39 & 2.26 & 92.5 & 377.0 & 4.12 & 5.41 \\
\hline 24 & GE- 3454 & 82 & 266.3 & 665.6 & 0.40 & 350.4 & 0.76 & 70.5 & 92.4 & 3.72 & 2.40 & 3.31 & 135.5 & 643.3 & 4.75 & 5.81 \\
\hline 25 & GE-3457 & 82 & 221.9 & 595.6 & 0.37 & 308.0 & 0.73 & 86.9 & 93.1 & 3.31 & 2.70 & 3.56 & 146.0 & 516.7 & 3.54 & 5.31 \\
\hline 26 & GE-4248 & 79 & 362.9 & 678.4 & 0.53 & 425.4 & 0.86 & 91.0 & 124.4 & 3.37 & 3.20 & 2.32 & 91.4 & 419.8 & 4.61 & 5.39 \\
\hline 27 & GE-4711 & 82 & 199.3 & 534.3 & 0.37 & 249.7 & 0.80 & 38.6 & 37.5 & 6.66 & 1.97 & 1.42 & 58.2 & 250.9 & 4.38 & 5.84 \\
\hline 28 & GE- 4736 & 83 & 252.1 & 673.1 & 0.38 & 326.1 & 0.78 & 52.8 & 60.8 & 5.36 & 2.59 & 2.21 & 91.7 & 367.9 & 4.03 & 5.65 \\
\hline 29 & GE-4738 & 82 & 222.6 & 512.2 & 0.44 & 283.4 & 0.79 & 52.1 & 56.6 & 5.02 & 1.90 & 2.86 & 117.3 & 508.5 & 4.34 & 5.33 \\
\hline 30 & GE- 4777 & 82 & 230.2 & 530.8 & 0.43 & 285.4 & 0.81 & 50.7 & 50.7 & 5.71 & 2.03 & 1.80 & 73.6 & 312.3 & 4.27 & 5.57 \\
\hline 31 & GE-4823 & 82 & 216.4 & 495.5 & 0.44 & 295.5 & 0.73 & 93.4 & 105.6 & 2.81 & 2.27 & 2.22 & 90.8 & 368.8 & 4.05 & 5.67 \\
\hline 32 & GE-4999 & 94 & 177.5 & 569.9 & 0.31 & 244.1 & 0.73 & 81.6 & 78.8 & 3.17 & 1.69 & 2.11 & 98.9 & 493.7 & 5.00 & 5.08 \\
\hline 33 & GE-5192 & 83 & 225.4 & 579.4 & 0.39 & 270.2 & 0.84 & 45.2 & 44.1 & 6.08 & 2.14 & 1.89 & 78.2 & 359.0 & 4.59 & 5.50 \\
\hline \multicolumn{2}{|c|}{ Mean (LD) } & 83 & 245.4 & 597.5 & 0.41 & 312.6 & 0.79 & 65.8 & 75.8 & 4.49 & 2.33 & 2.42 & 99.7 & 416.5 & 4.22 & 5.49 \\
\hline \multicolumn{2}{|c|}{ Grand Min. } & & 160.1 & 434.0 & 0.31 & 225.7 & 0.66 & 38.5 & 37.5 & 2.12 & 1.39 & 1.31 & 35.5 & 249.9 & 2.98 & 4.98 \\
\hline \multicolumn{2}{|c|}{ Grand Max. } & & 378.5 & 895.2 & 0.53 & 495.1 & 0.86 & 95.1 & 201.0 & 6.66 & 3.39 & 3.63 & 145.8 & 654.7 & 8.27 & 6.53 \\
\hline \multicolumn{2}{|c|}{ Grand Mean } & & 261.5 & 624.9 & 0.42 & 330.6 & 0.79 & 67.6 & 84.0 & 4.36 & 2.52 & 2.35 & 88.9 & 428.6 & 5.04 & 5.58 \\
\hline \multicolumn{2}{|c|}{$\operatorname{SEm} \pm$} & & 37.5 & 72.2 & 0.02 & 48.7 & 0.02 & 7.48 & 9.2 & 0.44 & 0.04 & 0.20 & 7.4 & 27.1 & 0.32 & 0.27 \\
\hline \multicolumn{2}{|c|}{$\mathrm{CD}(\mathrm{P}<0.05)$} & & 103.9 & 200.0 & 0.05 & 134.9 & 0.05 & 20.7 & 25.4 & 1.21 & 0.11 & 0.55 & 20.5 & 75.1 & 0.89 & 0.74 \\
\hline \multicolumn{2}{|c|}{ CV (\%) } & & 20.3 & 16.3 & 6.5 & 20.8 & 2.9 & 15.7 & 15.4 & 14.2 & 2.8 & 12.0 & 11.7 & 9.0 & 9.1 & 6.8 \\
\hline
\end{tabular}


Table.2 Trait specific accessions/ varieties of finger millet

\begin{tabular}{|c|c|c|c|c|}
\hline Trait & $\begin{array}{l}\text { GPU-28 } \\
\text { (Check) }\end{array}$ & $\begin{array}{c}\text { CD @ } \\
5 \%\end{array}$ & Better accessions & Significance \\
\hline Grain yield & $328.8 \mathrm{~g} \mathrm{~m}^{2}$ & 103.9 & Indaf-9, GE-1013, MR-6, GE-4248 & NS \\
\hline Productive tillers & $63.9\left(\right.$ No.m $\left.{ }^{2}\right)$ & 20.7 & GE-224, GE-4823, GE-619, GE-162, GE-4248, GE-4222 & * \\
\hline No. of earheads & $108.4\left(\right.$ No.m $\left.^{2}\right)$ & 25.4 & GE-619, GE-224, GE-4248 & * \\
\hline Threshing percent & 0.77 & 0.05 & GE-4222, PR-202, GE-4248 (> $86 \%)$ & * \\
\hline Mean earhead wt. & 4.59 & 1.21 & Indaf-9, GE-1034, MR-6, GE-844, GE-4711, GE-5192 & $*$ \\
\hline 1000 seed weight & $3.39 \mathrm{~g}$ & 0.11 & GPU-28 & \\
\hline Biomass at harvest & $790.6 \mathrm{~g} \mathrm{~m}^{2}$ & 200.0 & Indaf-9 and GE-619 (> 880 g.m2) & NS \\
\hline Harvest index & 0.41 & 0.05 & VR-708, GE-1013, L-5, GE-4248 & $*$ \\
\hline LAI & 2.97 & 0.55 & GE-619, GE-3457 & * \\
\hline LAD & 110.0 days & 20.5 & GE-619, GE-3457 & $*$ \\
\hline Biomass at flowering & $623.8 \mathrm{~g} \mathrm{~m}^{2}$ & 75.1 & GE-619, GE-3454 & NS \\
\hline DM/LAD & $5.68 \mathrm{~g} \mathrm{~m}^{2} \mathrm{~d}^{-1}$ & 0.89 & GE-4732, GPU-48, Indaf-9, VR-708, GE-1034 & $*$ \\
\hline SLW & $6.53\left(\mathrm{mg} \mathrm{cm}^{2}\right)$ & 0.78 & GPU-28 & \\
\hline
\end{tabular}


Table.3 Rainfall and soil temperature during crop growth period

\begin{tabular}{|c|c|c|c|}
\hline Stage of the crop & $\begin{array}{c}\text { Short } \\
\text { Duration }\end{array}$ & $\begin{array}{l}\text { Medium } \\
\text { duration }\end{array}$ & $\begin{array}{c}\text { Long } \\
\text { duration }\end{array}$ \\
\hline \multicolumn{4}{|l|}{ (1) 15 days prior and to Flag leaf stage } \\
\hline (a) Rainfall (mm) & $100(9)$ & $137.5(9)$ & $60.1(5)$ \\
\hline (b) Soil temperature $\left(0^{c}\right)$ at $10 \mathrm{~cm}$ soil depth & 23.2 & 26.2 & 27.3 \\
\hline \multicolumn{4}{|l|}{ (2) Flag leaf to Ear head emergence stage } \\
\hline (a) Rainfall (mm) & $86.3(6)$ & $6.4(1)$ & $\mathbf{0}$ \\
\hline (b) Soil temperature $\left(0^{c}\right)^{c}$ at $10 \mathrm{~cm}$ soil depth & 26.2 & 27.9 & 30.2 \\
\hline \multicolumn{4}{|l|}{ (3) Ear head emergence stage to $50 \%$ flowering } \\
\hline (a) Days to $50 \%$ flowering & 62 & 74 & 83 \\
\hline (b) Rainfall (mm) & $6.4(1)$ & 0 & $\mathbf{0}$ \\
\hline (c) Soil temperature $\left(0^{c}\right)$ at $10 \mathrm{~cm}$ soil depth & 27.8 & 29.3 & 36.1 \\
\hline \multicolumn{4}{|l|}{ (4) $50 \%$ flowering to $50 \%$ grain filling stage } \\
\hline (a) Rainfall (mm) & 0 & $43.2(1)$ & $144.6(9)$ \\
\hline (b) Soil temperature $\left(0^{c}\right)$ at $10 \mathrm{~cm}$ soil depth & 29.1 & 35.3 & 28.9 \\
\hline
\end{tabular}

Note: (1) Date of sowing, 03-08-2007

(2) No rainfall during $21^{\text {st }}$ September to $18^{\text {th }}$ October, 2007

(3) Values in parenthesis indicates number of rainy days

Table.4 Relationship between physiological, growth and yield attributes in finger millet genotypes

\begin{tabular}{|c|c|c|c|c|c|c|c|c|c|c|c|c|c|c|}
\hline 1 & 2 & 3 & 4 & 5 & 6 & 7 & 8 & 9 & 10 & 11 & 12 & 13 & 14 & 15 \\
\hline Parameters & DFF & TDMH & HI & Straw wt & Thr \% & Ear wt. & $\begin{array}{l}\text { Ear } \\
\text { No. }\end{array}$ & PT No. & $\begin{array}{l}1000 \text { seed } \\
\text { wt. }\end{array}$ & LAI & LAD & TDMF & DM/LAD & SLW \\
\hline 1) Grain yield & -0.21 & $0.87 * *$ & $0.52 * *$ & $0.58 * *$ & $0.41^{*}$ & $0.95 * *$ & 0.30 & 0.12 & $0.69 * *$ & 0.32 & 0.19 & $0.43^{*}$ & 0.21 & 0.23 \\
\hline $\begin{array}{l}\text { 2) Biomass at } \\
\text { harvest (TDMH) }\end{array}$ & & & 0.05 & $0.88 * *$ & 0.12 & $0.90 * *$ & 0.32 & 0.12 & $0.45^{* *}$ & $0.41 *$ & 0.31 & $0.54 * *$ & 0.15 & 0.11 \\
\hline 3) $\mathrm{HI}$ & & & & $-0.34 *$ & $0.63^{* *}$ & $0.38^{*}$ & 0.10 & 0.04 & $0.66 * *$ & -0.06 & $\begin{array}{c}- \\
0.16\end{array}$ & -0.04 & 0.19 & 0.30 \\
\hline 4) Straw weight & & & & & 0.09 & $0.59 * *$ & 0.23 & 0.04 & 0.13 & 0.32 & 0.27 & $0.42 *$ & 0.09 & -0.12 \\
\hline 5)Threshing \% & & & & & & 0.12 & $\begin{array}{c}- \\
0.10\end{array}$ & -0.22 & $0.42 *$ & -0.24 & $\begin{array}{c}- \\
0.32\end{array}$ & -0.21 & 0.22 & -0.03 \\
\hline 6) Ear weight & & & & & & & $\begin{array}{c}0.34 \\
*\end{array}$ & 0.16 & $0.64 * *$ & $0.41 *$ & 0.29 & $0.53 * *$ & 0.18 & 0.30 \\
\hline 7) Earhead number & & & & & & & & $\begin{array}{l}0.80^{*} \\
*\end{array}$ & 0.22 & $0.41 *$ & 0.29 & $0.36^{*}$ & 0.01 & -0.23 \\
\hline $\begin{array}{l}\text { 8) Productive tiller } \\
\text { number }\end{array}$ & & & & & & & & & 0.01 & 0.31 & 0.24 & 0.21 & -0.10 & -0.30 \\
\hline 9) 1000 seed weight & & & & & & & & & & 0.16 & 0.02 & 0.23 & 0.31 & $\begin{array}{c}0.43 \\
*\end{array}$ \\
\hline 10) LAI & & & & & & & & & & & $\begin{array}{c}0.92 \\
* *\end{array}$ & $0.84 * *$ & -0.30 & -0.29 \\
\hline 11) LAD & & & & & & & & & & & & $0.71 * *$ & $-0.57^{* *}$ & $\begin{array}{c}- \\
0.34 \\
*\end{array}$ \\
\hline $\begin{array}{l}\text { 12) Biomass at } \\
\text { flowering (TDMF) }\end{array}$ & & & & & & & & & & & & & 0.13 & -0.10 \\
\hline 13) DM/ LAD & & & & & & & & & & & & & & 0.41 \\
\hline
\end{tabular}

$*(0.34)$ and $* *(0.44)$ represents significance at $5 \%$ and $1 \%$ respectively 
Therefore, an increase in LAI would lead to increased biomass and grain yield especially in medium duration varieties in the changing climate scenario. The accessions GE-1034, GE-4222 (short duration), GE-1013, GE-619 (medium duration) and GE-4248 (long duration) possessing high leaf area with moderate to high assimilation rates would serve as donors in breeding programmes, possibly to break yield plateau of finger millet.

\section{References}

Anonymous, 2011. Annual Report, All India Coordinated Small Millets Improvement Project, GKVK, Bangalore 65, Karnataka.

Aparna, K. and Ansari, Z.G., 2017. Evaluation of ragi genotypes on growth parameters and physiological attributes under kharif rainfed conditions. International Journal of Chemical Studies. 5(6): 1899-1901.

Bedis, M.R., Patil, H.S., Jangle, G.D. and Patil, V.S., 2006. Correlation and path analysis in finger millet (Eleusine coracana (L) Gaertn). Crop Research. 31: 264-266.

John, S. and Kumar, P., 2018. Character association among vegetative, preyield and yield parameters in finger millet (Eleusine coracana L.). International Journal of Pure and Applied Science. 6(2): 156-161.

Khalak, A. and Kumaraswamy, A.S., 1994. Effect of irrigation and residues of fertilizer levels on performance of finger millet in potato-based cropping system. Mysore Journal of Agricultural Sciences. 28: 111-115.

Kumar, Y.K.D., Vishwanath, A.P., Ananda, M.R., Vittal Navi and Rehaman, H.M.A., 2006. Correlation and regression studies in finger millet (Eleusine coracana L.). Environment and Ecology. 4: 1050-1052.

Kumara. O., Basavaraj Naik, T. and Palaiah, P., 2007. Effect of weed management practices and fertility levels on growth and yield parameters in finger millet. Karnataka Journal of Agricultural Sciences. 20: 230-233.

Malhotra, S.K., 2018. National conference on agriculture: Kharif campaign, 2018. www.agricoop.gov.in/sites/default/file s/agriculture_commissioner_ppt_1.ppt $\mathrm{X}$.

Nandini, B., Ravishankar, C.R., Mahesha, B., Shailaja, H. and Kalyana Murthy, K.N., 2010. Study of correlation and path analysis in $F_{2}$ population of finger millet. International Journal of Plant Science. 5: 602-605.

Narayan, H., Ramachandrappa, B.K., Mudalairiyappa and Thimme Gowda., M.N., 2018. Yield and economics of finger millet with establishment methods under different planting geometry and nutrient source. Indian Journal of Dryland Agricultural Research and Development. 33(1): 5458.

Richards, R.A., Condon, A.G. and Rebetzke, G.J., 2001. Traits to improve yield in dry environments. In: M.P. Reynolds, J.I. Ortiz-Monasterio \& A. McNab (Eds.), Application of Physiology in Wheat Breeding, pp. 88-100. CIMMYT, Mexico

Richards, R.A., 2000. Selectable traits to increase crop photosynthesis and yield of grain crops. Journal of Experimental Botany. 51: 447-458.

Roy, D.K., Chakraborty, T., Sounda, G. and Maitra, S. 2002. Growth and yield attributes of finger millet as influenced by plant population and different levels of nitrogen and phosphorus. Indian Agriculturist. 46: 65-71.

Shankar, A.G., Udaya Kumar, M. and Prasad, T.G., 1990. Genetic variability for net 
photosynthesis in finger millet (Eleusine coracana L.) genotypes An approach to identify high CER types. Journal of Agronomy and Crop Science. 165: 240-252.

Sharathbabu, K.S., Shanthakumar, G. and Salimath, P.M., 2008. Genetic variability and character association in white ragi (Eleusine coracana (L.) Gaertn.). Karnataka Journal of Agricultural Sciences. 21: 572-575.

Siebke, K., Ghannoum, O., Conroy, J.P., Badger, M.R. and Von Caemmerer, S., 2003. Photosynthetic oxygen exchange in $\mathrm{C}_{4}$ grasses: the role of oxygen as electron acceptor. Plant Cell and Environment. 26: 1963-1972.

Subrahmanyam, D., 2000. Genotypic variability in photosynthetic characters in finger millet. Photosynthetica. 38: 105-109.

Sujatha, D. and Rao, J.S.P., 2003. Influence of bio-regulators on growth, nutrient utilization efficiency and yield of finger millet (Eleusine coracana L.). Journal of Research ANGRAU. 31: 31-38.

Swetha, T.N., 2011. Assessment of the contribution of phylogical traits to grain yield during crop improvement of finger millet (Eleusine coracana) M. Sc (Agri.) thesis, Department of Crop Physiology, University of Agriculture Sciences., Bangalore-65.

Udayakumar, M., Sashidhar, V.R. and Prasad, T.G., 1986. Physiological approaches for improving productivity of finger millet under rainfed conditions. In: Seetharam, A., Riley, K.W. and Harinarayana, G. (eds.), Small millets in global agriculture, pp, 200228. Oxford \& IBH Publishing Co.

Uma, M.S., 1987. Transpiration quotient and water use efficiency in different $\mathrm{C}_{3}$ and $\mathrm{C}_{4}$ species and its relationship with biomass and productivity under moisture stress conditions. M.Sc. (Agri.) Thesis, University of Agricultural Sciences, Bangalore.

Ueno, O., Kawano, Y., Wakayama, M. and Takeda, T., 2006. Leaf vascular systems in $\mathrm{C}_{3}$ and $\mathrm{C}_{4}$ grasses: $\mathrm{A}$ two dimensional analysis. Annals of Botany. 97: 611-621.

Veeraputhiran, R., Chellamuthu, V. and Pandian, B.J., 2009. Performance of finger millet varieties in coastal region of Karaikal. International Journal of Agricultural Sciences. 5(1): 190-192.

Vishwanath, K.A., 2005. Genetic variability in root traits and WUE in finger millet: Assessment of contrast in these traits in water limited condition. M.Sc., (Agri.) theis, Department of Crop Physiology, University of Agricultural Sciences, GKVK, Bangalore, Karnataka, India.

Wolie, A. and Dessalegn, T., 2011. Correlation and path coefficient analysis of some yield related traits in finger millet (Eleusine coracana (L.) Geartn.) genotypes in north-west Ethiopia. African Journal of Agricultural Research. 6: 5099-5105.

\section{How to cite this article:}

Nanja Reddy, Y.A., Jayarame Gowda, E.G. Ashok, K.T. Krishne Gowda and Gowda, M.V.C. 2019. Higher Leaf Area Improves the Productivity of Finger Millet (Eleusine coracana (L.) Gaertn) under Rainfed Conditions. Int.J.Curr.Microbiol.App.Sci. 8(05): 1369-1377. doi: https://doi.org/10.20546/ijcmas.2019.805.156 